\title{
La musique traditionnelle face à la maladie et à la possession chez les Touaregs de l'Ahaggar (Sud de l'Algérie)
}

Faiza Seddik-Arkam

\section{(2) OpenEdition \\ Journals}

Édition électronique

URL : http://journals.openedition.org/ethnomusicologie/110

ISSN : 2235-7688

Éditeur

ADEM - Ateliers d'ethnomusicologie

Édition imprimée

Date de publication : 1 novembre 2006

Pagination : 139-159

ISSN : 1662-372X

Référence électronique

Faiza Seddik-Arkam, « La musique traditionnelle face à la maladie et à la possession chez les

Touaregs de l'Ahaggar (Sud de l'Algérie) », Cahiers d'ethnomusicologie [En ligne], 19 | 2006, mis en ligne le 15 mars 2013, consulté le 19 avril 2019. URL : http://journals.openedition.org/ ethnomusicologie/110

Ce document a été généré automatiquement le 19 avril 2019

Tous droits réservés 


\section{La musique traditionnelle face à la maladie et à la possession chez les Touaregs de l'Ahaggar (Sud de l'Algérie)}

Faiza Seddik-Arkam

\section{Les Touaregs Kel Ahaggar face à la modernité}

1 La société touarègue Kel Ahaggar actuelle a hérité des échanges socioculturels, politiques et économiques avec les populations du nord et du sud du Sahara. Les échanges commerciaux transsahariens, l'élevage pastoral et le trafic caravanier ayant marqué son histoire, elle a, par la suite, subi les effets de la colonisation. Des frontières arbitraires ont été établies durant cette période et elles ont été maintenues après les indépendances. La fin des rezzous et la quasi disparition du trafic caravanier ont miné l'économie touarègue. Les Touaregs qui nomadisaient dans ces territoires du Sahara et du Sahel ont vu leur espace se rétrécir considérablement.

Pour ce qui concerne les Kel Ahaggar, des changements significatifs ont été apportés par l'accession du pays à l'indépendance et, notamment, par l'introduction du salariat, la scolarisation des enfants, la sédentarisation et les coopératives agricoles. La société des Kel Ahaggar a été marquée, depuis le début des années soixante-dix, par une série de bouleversements dus au contact avec l'économie moderne et à la dégradation de l'environnement naturel. La sécheresse et, par la suite, la répression ont provoqué un brusque afflux de « réfugiés » touaregs du Niger et du Mali vers Tamanrasset et les autres centres frontaliers.

Cette société tente tant bien que mal de s'adapter physiquement et psychiquement aux nouvelles conditions de vie amenées par la sédentarité. Et pour ne pas se laisser dépérir, elle crée de nouveaux mécanismes de défense inspirés d'un système magico-religieux et thérapeutique qui prend son origine dans la culture et les croyances ancestrales 
construisant sa cosmogonie, ainsi que dans les traditions islamiques, qui se sont adaptées à cet univers magico-religieux.

4 Chez les Touaregs, ce sont les Kel essuf (les " génies de la solitude ») qui alimentent cette croyance bien antérieure à l'islam, orientant une véritable cosmogonie. Cette croyance entraîne une série de comportements, d'attitudes mentales qui marquent profondément le psychisme des individus et leurs relations dans les structures familiales elles-mêmes (Gast 1985). L'essuf est le domaine des génies, des démons et des ancêtres ; c'est en ce sens qu'il diffère des djinns arabes, aljinnan en tamahaq (langue touarègue de l'Ahaggar).

5 Ce système de croyances est lui-même en pleine recomposition car il subit progressivement l'influence de divers apports culturels. L'intégration de certains rituels venus d'ailleurs, lors des plus importantes cérémonies, en est le signe.

6 La ville de Tamanrasset est actuellement investie par de nombreuses communautés de différentes origines qui se sont installées progressivement dans la ville et à sa périphérie, et qui en ont modifié le paysage socioculturel et les structures locales. Les nomades touaregs se retrouvent alors minoritaires dans un ensemble pluriculturel et pluriethnique. La sédentarisation induit aussi une cohabitation avec les villageois, un changement de travail, de régime alimentaire et d'habitat, mais aussi de nouvelles influences sur le plan culturel, notamment musical.

\section{Les transformations de l'espace musical traditionnel}

7 Les modes de transmission du savoir oral se trouvant affectés, l'influence des médias et de la radio ont beaucoup joué dans l'étouffement et le repli de certains éléments culturels, caractéristiques des Touaregs. Les expressions culturelles se laissent progressivement dominer par la folklorisation encouragée par les politiques locales, qui voient là un attrait pour les touristes. Les occasions qui réunissaient régulièrement la communauté touarègue autour des assemblées musicales et poétiques se font rares. Les rituels traditionnels qui avaient lieu autour des événements les plus importants de la vie ne trouvent plus d'espace adéquat. Ils s'adaptent et évoluent dans un espace différent, lorsqu'ils ne disparaissent pas complètement. Cette transformation est particulièrement sensible dans les formes artistiques de la tradition noble, telle que la pratique de la vièle monocorde imzad. L'un des soucis de la jeunesse touarègue actuelle est de maintenir des symboles culturels forts tels que la pratique musicale, la poésie et la danse chamelière lors des illugan (ronde des chameaux), qui retracent l'histoire d'un passé guerrier prestigieux.

8 On assiste également à une conquête des nouveaux espaces semi urbains, celle des quartiers périphériques de Tamanrasset où la communauté se réunit la nuit, organise des zahuten (de l'arabe zahu : divertissement, distraction), souvent au son du tambour ou de la guitare (Bellil et Dida 1993 : 106). C'est ainsi que des soirées galantes s'organisent autour de Lalla, la dame du tindi, dans le quartier de Tahaggart-choumara, réunissant les jeunes touaregs autour du tambour-mortier tindi. Lors de ces retrouvailles, il y a toujours un sévère code de conduite à respecter.

9 Dans ces quartiers ont aussi lieu régulièrement des tazengharet qui entraînent des possessions, et des khomissa, rituels musicaux venant du Niger et menés traditionnellement par des enaden (forgerons). Autour d'une scène, des hommes et des 
femmes se tiennent face à face, dans des danses très suggestives. Ces danses mixtes provoquent régulièrement des «transes » (agelled).

Lorsqu'on se promène aujourd'hui dans ces quartiers la nuit, on est vite attiré par la multitude des bruits provenant des nombreuses fêtes et cérémonies musicales organisées par des jeunes, mêlant la fête, l'odeur des encens, le son du tambour à la «transe » des corps. La proximité physique favorise le mélange des espaces féminins et masculins. Ces danses et ces musiques réunissent des jeunes d'horizons différents, parmi lesquels des ichoumar, réfugiés touaregs du Niger et du Mali qui voyagent beaucoup et qui fêtent ainsi leur retour.

Un nouveau style dit « al guitara ", introduit par la jeunesse de l'Adagh, s'est répandu très rapidement parmi les Kel Ahaggar. Grâce à cette nouvelle forme musicale, l'artiste s'individualise, il s'accompagne à la guitare et chante des textes subversifs, remettant en cause l'ordre établi, mais il chante aussi les amours impossibles. D'autres fêtes nocturnes sont organisées en ville, réunissant une population hétéroclite: jeunes militaires désœuvrés, prostituées... faisant de cette ville de passage l'espace de toutes les transgressions, notamment celles des règles de l'asshak (pudeur, honneur), qui opère une mutation significative dans la société touarègue.

C'est dans un contexte de violation de l'espace désertique sacré avoisinant la ville que l'on observe une adaptation du rituel à l'espace urbain. Jadis réservé au monde de l'essuf qui avait ses frontières symboliques, cet espace est violé par de nouvelles constructions. Les crues des oueds, qui emportent les gens sur leur passage, seraient une manifestation des Kel essuf (les " génies de la solitude ») qui y résident, principalement en raison de la colère des saints, qui n'ont pas voulu protéger ces quartiers, et des représailles des génies qui y habitent. Les maladies causées par les génies sont clairement des désordres liés à l'essuf, à la solitude de la brousse où règnent des forces redoutables; elles sont aussi souvent reliées à la sorcellerie. La victime perd son comportement social, elle ne parle plus ; plongée dans l'hébétude la plus totale, elle rejette son entourage et s'enferme dans le silence. Les malades situent toujours leur trouble à la suite d'événements traumatisants : peurs, rencontres effrayantes, cauchemars... Pour tenter de ramener la victime à la rencontre de ses semblables, au sein de sa communauté, la thérapeutique appliquée consiste en premier lieu à lui faire écouter de la musique chantée.

\section{La divination et le recours à la musique}

13 Un riche vocabulaire concerne les différentes manières dont peuvent intervenir les Kel essuf. Une personne peut être vue comme adessentu, pénétrée par les Kel essuf, ou alors mamsoussa, qui, en arabe, veut dire «touchée par les génies ». Une autre est seulement "visitée par les génies" (zarent Kel essuf). Dans de nombreux cas, cela nécessite une séance de musique de possession de tindi ou de tazengharet.

14 Un devin pose le diagnostic et désigne l'origine ou la cause du mal, mais il ne détaille pas et n'offre pas de solution au malade. Il permet l'identification du mal sans le nommer explicitement. Par contre, il peut reconnaître la nature du Kel essuf mis en cause, et de ce fait diriger vers un officiant de culte de possession ou vers tout autre spécialiste traditionnel de l'invisible. A partir de ce diagnostic, plusieurs recours sont possibles; parmi eux la séance de musique rituelle. 
La perte de parole est interprétée comme une conséquence d'une attaque des Kel essuf, l'expression touarègue wattent Kel essuf, veut dire que cette personne a été «frappée par les génies ", madrûb (m), madrûba (f) en arabe, et qu'elle nécessite un exorcisme. Certaines cérémonies musicales ont pour objectif de chasser les génies, lorsque ces derniers sont à l'origine d'un profond mal-être chez une personne. Et il y a un stade critique de la possession qui est l'anebzug, la folie : le malade est littéralement dévoré par les Kel essuf, il est coupé de la société, se déshabille et s'enfuit en brousse. Pour éviter qu'il ne se fasse du mal ou qu'il soit violent avec les autres, le malade est parfois ligoté avant d'être confié à un taleb. Actuellement, il est aussi parfois amené en service de psychiatrie lors de crises violentes.

16 L'état du madrûb, du «frappé », peut nécessiter des méthodes plus radicales, telles que l'exorcisme pratiqué lors des rites de possession religieux et thérapeutiques, que l'on nomme tamagrawt en tamahaq, qui se réalise par le biais de l'imposition des mains de la part d'un lettré ou de rokia (en arabe "incantation », " désenvoûtement ») par le biais du souffle et du Coran. Ces deux procédés rituels sont pratiqués par les tolba. Certains d'entre eux feront appel au sacrifice d'un animal (izni), indispensable au rituel, nommé selon les circonstances sedqa ou fedya en arabe et takute en tamahaq.

\section{Musique et thérapie : le cas de la vièle monocorde imzad}

17 En Ahaggar, certains genres musicaux sont en voie de disparition et ne gardent que très peu de pratiquants. Mais ceci concerne essentiellement le répertoire de l'imzad: «il apparait d'autre part que le mouvement de religiosité assez intense qui accompagne la "pacification" de l'Ahaggar avec l'arrivée de tolba et chorfa venus du Nord joue indirectement un rôle dans la disparition progressive de certains genres musicaux » (M écheri-Saada 1996 : 33). Durant la période coloniale, déjà, certains auteurs ajoutent que « dans certains campements [...] l'imzad a été rigoureusement interdit, car les nouveaux puritains lui attribuent une influence licencieuse sur la jeunesse» (Balout 1959: Pl. LXXV).

18 Sur l'air d'imzad joué par une femme, un homme chante des poèmes, et seul le son de sa voix accompagne l'instrument. L'ambiance est alors à la séduction et à la poésie. La séduction de la vièle et les cours d'amour, lors des assemblées poétiques autour de l'ahal, inquiétaient les puritains, qui étaient sous l'influence des groupes religieux de chorfa qui se sont introduits durant cette période dans la société touarègue. L'imzad, intimement associé aux poésies guerrières et aux chants d'amours, a perdu ses thèmes. Mais la créativité poétique demeure vive, elle s'accompagne de guitare et de luth, instruments introduits par l'échange culturel avec les styles des autres régions et des pays voisins. L' imzad demeure néanmoins l'instrument emblématique de toute une culture.

19 Une autre fonction de la musique de l'imzad semble exister depuis longtemps en Ahaggar. C'est celle d'accompagner des cérémonies d'exorcisme. Mais cette fonction s'observe de plus en plus rarement, peut-être parce que le maintien de l'imzad en général pose actuellement problème, bien qu'il soit considéré comme l'élément le plus prestigieux de la culture touarègue. Chez les Touaregs de l'Aïr, on continue cependant de jouer de l' anzad (anzad ou tindi n gumaten) pour " chasser les génies de la tête du malade » (Borel $1986: 112)$. 
20 La poésie chantée associée à l'imzad était le genre poétique par excellence de la noblesse touarègue traditionnelle : «Aussi bien le contenu sémantique que le cadre d'exécution de ces chants reflètent le mode de vie et l'éthique de l'ancienne classe dominante " (Mécheri-Saada 1986: 96). La structure hiérarchique se reflète aussi dans la vie musicale, où elle s'accompagne d'une différenciation sexuelle. Aux hommes le chant en solo ou accompagné, composé d'un répertoire classique se rapportant aux prouesses guerrières, souvent créé par les héros guerriers, et aux femmes des catégories suzeraines ou tributaires de reproduire ces airs instrumentaux sur la vièle monocorde imzad. La maîtrise de cet instrument semble s'être élargie à d'autres catégories sociales : « La classification hiérarchique idéale est sujette à de nombreuses exceptions [...]. La continuité des pratiques sociales qui garantissent son identité touarègue est donc fortement compromise » (Borel $1988: 28$ ).

21 Actuellement, en Ahaggar, l'une de celles qui maîtrisent le mieux les airs de l'imzad - et l'une des dernières gardiennes de cet art musical ancestral - est Khaoulen, une femme de la catégorie des forgerons enaden qui est sollicitée à chaque grande occasion. Nous avons eu le loisir d'observer la pratique de l'imzad dans un contexte différent de celui qui lui est traditionnellement assigné. Ce fut au cours d'un pèlerinage religieux, à proximité du village de Tarhananet, que j'ai écouté des airs d'imzad accompagnant exceptionnellement une tazengharet qui se tenait à proximité. Dans ce cas précis, l'imzad semblait avoir réellement une fonction thérapeutique. Cette tazengharet, qui a vu des hommes et des femmes tomber en catalepsie, avait tout l'aspect d'un rituel de possession. Lors de cette cérémonie, un jeune homme, décrit comme possédé (igullel) par ses pairs, s'était distingué par un comportement étrange. Il s'était éloigné du cercle de danse et s'était rapproché de Khaoulen, la musicienne qui était assise à proximité et jouait des airs d'imzad. 
Fig. 1 : Khaoulen, célèbre joueuse d'imzad du groupe enaden (forgerons), lors d'une manifestation musicale. Tamanrasset.

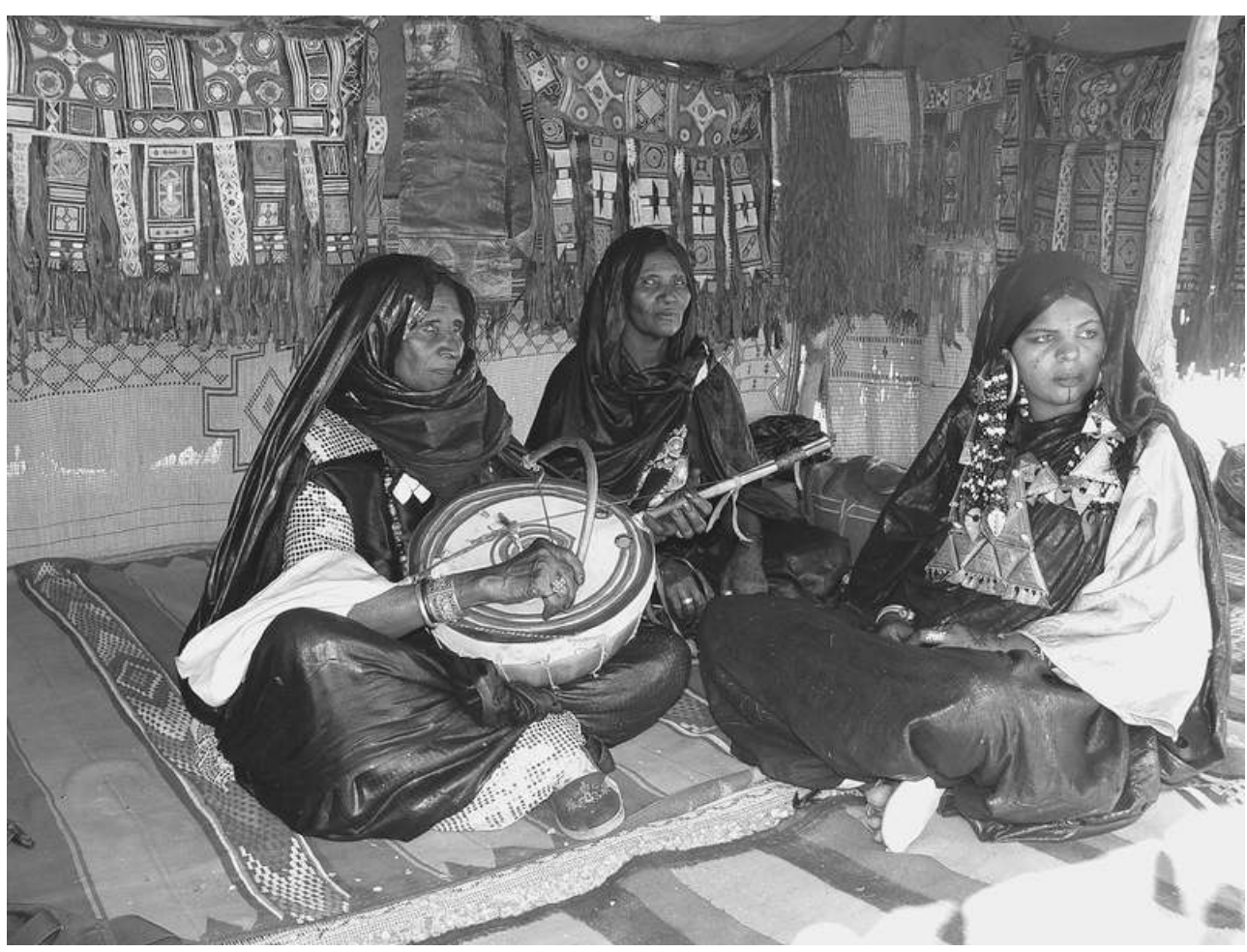

Photo association « Sauver l'imzad », avril 2005.

Assise et tenant fermement l'instrument, la musicienne jouait différents airs en retrait de la scène; elle adaptait progressivement son jeu à l'état et à la gestuelle du possédé, jusqu'à trouver le rythme qui lui correspondait. Elle portait une très grande attention à l'évolution de son état. Ce dernier, tout en effectuant des gestes désordonnés, s'approchait d'elle en tapant des pieds sur le sol (rukud), provoquant un brouillard de sable, la menaçant de son bâton, et exigeant d'elle, avec des cris de fureur et d'une voix inaudible, de changer de rythme. La musicienne, qui semblait habituée à ce genre de réactions, gardait sa sérénité, arrêtait son jeu le temps qu'il s'éloigne, et reprenait de plus belle en changeant de rythme au fur et à mesure, accompagnant ainsi sa danse. Les rythmes de l'imzad changeaient en fonction de l'évolution de l'état de cet homme en transe. Le jeune possédé tournoyait, l'air perdu et hagard (yeghlel), jusqu'à tomber en catalepsie. On a dit alors de lui qu'il était «iswa », qu'il avait bu, qu'il avait été arrosé par les chants et la musique. Il s'agissait bien dans ce cas de rythmes joués pour les génies, et ces rythmes, joués à proximité d'une tazengharet qui, habituellement, n'introduit pas de musique instrumentale, n'accompagnaient aucun chant particulier lié aux thèmes traditionnels de l'imzad.

23 Ces rythmes seraient en fait de « devises musicales », comme chez les Songhay du Niger : les textes rituels sont chantés le plus souvent avec accompagnement instrumental (Rouch 1960 : 135), mais souvent aussi joués à la vièle sans être chantés, car ainsi ils «fournissent la substance même de la musique de possession » (Rouget 1988 : 193).

Mais force est de constater que cette maîtrise de l'imzad, n'est conservée que par quelques rares femmes initiées. Il se pose alors le problème de la transmission des savoirs dans le contexte actuel. Le poids des religieux ne semble pas peser outre mesure sur la pratique 
musicale, mis à part dans la disparition des cours de séduction de l'ahal. Les manifestations religieuses telles que les ziara (pèlerinages) sont l'occasion de nombreuses cérémonies musicales. Ces dernières voisinent avec des rituels soufis tels que le dhikr (a), ou azzeker $(\mathrm{t})$, et le chant mystique d'El Busri « el borda », que l'on retrouve également lors des mariages touaregs. C'est surtout l'évolution socio-économique et la transformation du mode de vie qui ont provoqué des mutations au sein de la société touarègue. Pour que ces musiques puissent encore se maintenir, il faudrait qu'elles retrouvent le rôle social qui faisait leur prestige, et le rôle magico-thérapeutique n'est pas des moindres.

\section{Le tindi}

Le tindi est une cérémonie musicale autour de poésies (tisiwal) chantées par des femmes touarègues ; il est centré sur un instrument de musique traditionnel, un tambour-mortier en bois appelé tindi, qui était jadis joué exclusivement par des femmes nobles. Cette pratique semble aujourd'hui s'être propagée au sein des autres catégories sociales ${ }^{1}$, et prend une forme magico-thérapeutique lorsqu'elle est organisée autour d'une femme possédée effectuant une danse assise (balancement de la tête et du corps). Ce rituel, qu'on observe de plus en plus rarement en Ahaggar, a lieu au sein du campement, sous une tente ou à l'extérieur de celle-ci, et ce au cours de la journée. Il se déroule dans un cercle familial très restreint. Le tambour y est joué pour les génies dans des cas de possession féminine. Le rituel est organisé pour une femme qu'on soupçonne être touchée par des Kel essuf et qui donne les signes d'une dépression, le plus souvent postnatale (fragilité psychique après un accouchement). Ce rituel concerne souvent les problèmes féminins liés à la sexualité et à la maternité. Dans ce cas, une femme va s'asseoir à proximité de la personne malade, jambes croisées, et frappe le tindi. Les femmes qui les entourent les accompagnent en battant des mains et en chantant, tandis que les hommes, debout, font un bruit de gorge appelé taxemxemt. La séance débute par une mélodie forte, syncopée, destinée à provoquer la transe (egalled), et dont le rythme rapide va amener le malade dans le monde des Kel essuf, « objet de ses peurs et de ses désirs » (Khawad 1979 : 81). Puis le rythme se ralentit, accompagné alors de poèmes tristes chantés par les femmes. Un peu plus tard, le thème mélodique change de nouveau pour s'achever sur des mélodies douces, décrivant un paysage joyeux de la vie sociale. Un dialogue se noue ainsi entre la malade et l'assemblée, facilitant le retour de la malade parmi les siens. Ce changement de rythmes correspond aux variations des rythmes destinés aux génies.

«Le tindi, c'est juste pour te réjouir, pour t'apporter du réconfort, te permettre de te socialiser de nouveau avec les autres", me disaient les femmes de l'Ahaggar. Le tindi permet donc la réintégration du malade dans le groupe, sa resocialisation.

Cette fonction thérapeutique du tindi est très importante chez les Touaregs du Niger (voir Rasmussen 1992), où elle concerne également les hommes. Hawad nous décrit ainsi l'une de ces cérémonie autour d'un tindi : «le malade vêtu de son costume de fête parfumé, muni d'un sabre afin de lutter contre les mauvais esprits, est amené au milieu du cercle formé par l'assemblée » (Khawad 1979: 80). Une cérémonie similaire semble se maintenir en Ahaggar dans un cercle très privé et dans quelques rares campements, mais elle concerne essentiellement les femmes ; les hommes victimes d'un mal similaire lié à l'essuf, sont, quant à eux, dirigés vers les marabouts. Par contre, on observe régulièrement des tindi lors des plus importantes manifestations musicales touarègues, souvent organisées autour des illugan, la ronde des chameaux. 
san Rasmussen, qui a étudié la possession féminine touarègue chez les Kel Ewey, se base sur les critères esthétiques de la danse. Les femmes nobles, dit-elle, touchées par l'infortune et l'invasion des génies, sont amenées à danser tout en s'imposant des critères esthétiques à respecter: "La danse de la tête, en tant que mouvement gracieux et contrôlé, et son trope central oscillant comme la branche d'un arbre encapsulent des symboles culturels essentiels afin de les rendre presque acceptables en termes esthétiques et symboliques parmi les nobles touaregs traditionnels. Cependant, ce mouvement laisse supposer que l'on est malade, ou seul, ou dans un état de sauvagerie ayant besoin d'exorcisme» (Rasmussen 1994 : 75). La danse associée au jeu du tindi est appelée tindi $n$ gumaten; ce rituel réunit l'ensemble de la société touarègue. L'auteure dit que le comportement d'une femme en transe théâtralise un "drame social », mettant en interaction une forme artistique et une expression sociale. L'exhibition des femmes est mal considérée chez les nobles touaregs, en particulier lors d'occasions où sont mélangées les différentes catégories sociales. La possession semble néanmoins contribuer à apaiser les conflits et réunir dans un même rituel des groupes traditionnellement opposés : nobles et esclaves, femmes et hommes, jeunes et vieux.

Fig. 2 : Jeunes femmes touarègues du groupe tributaire Kel Ahnet en tenue rituelle, portant un masque de protection lors d'un mariage dans un village sédentarisé à Indelleg. Tamanrasset.

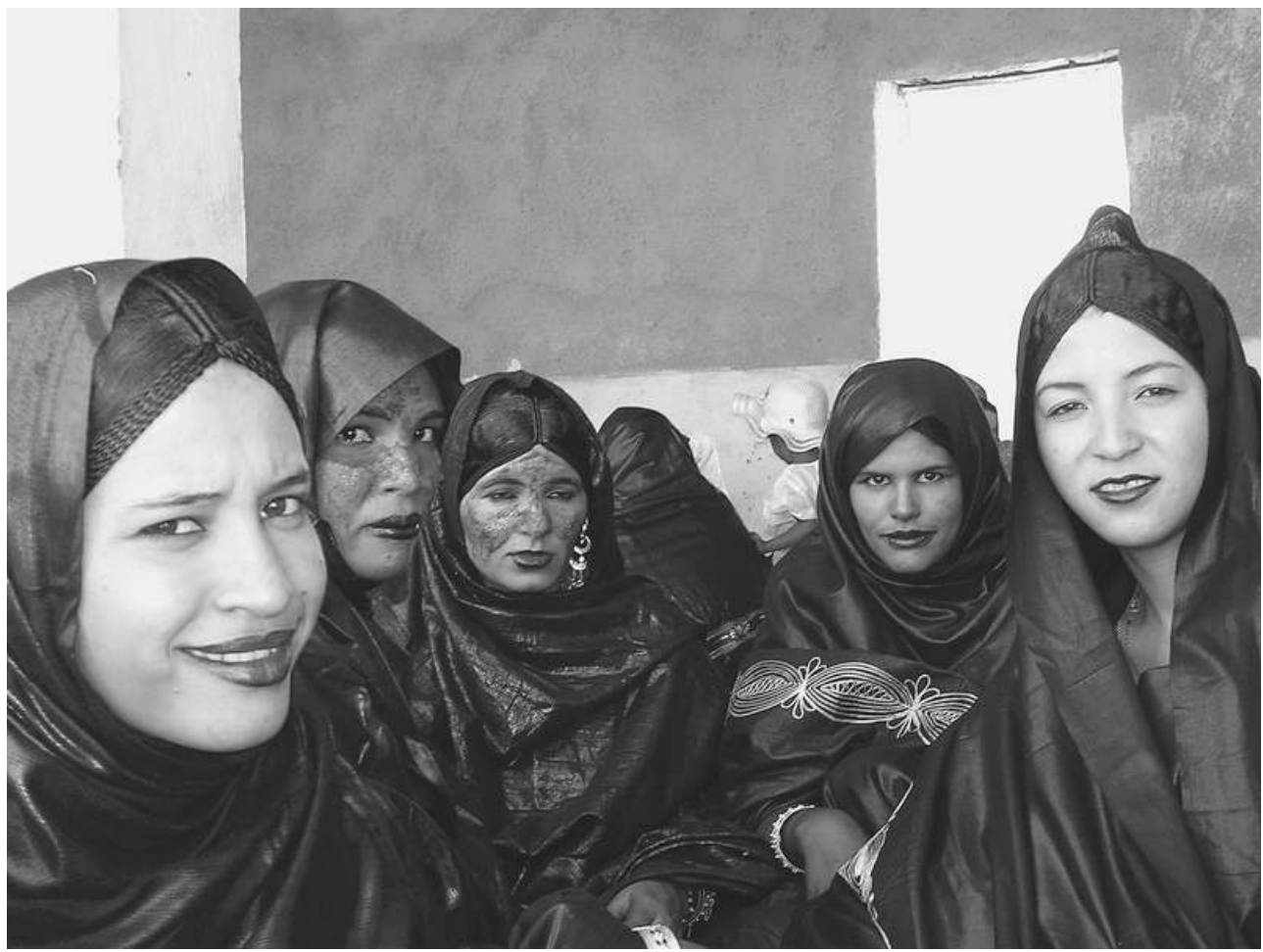

Photo Faiza Seddik-Arkam, août 2005.

La société accepte de voir une femme noble danser avec la tête et s'exhiber ainsi seulement si cela est justifié par l'état d'egalled. Ce mouvement est considéré comme strictement féminin; on dit que ce sont les esprits qui dansent en elle : «les femmes en état de transe transforment la culture soudanaise ", nous dit Rasmussen, elles exécutent un mouvement gracieux et contrôlé de la tête et du buste pour le faire accepter dans leur milieu noble : « La danse de la tête est un compromis élégant qui brouille la ligne entre la 
danse et la possession, qui prend des images acceptables dans des chansons et des façons de bouger appropriées » (Rasmussen 1994).

En principe, on ne chante ni ne joue d'un instrument à n'importe quel moment. Il existe des occasions spécifiques dont les deux plus importantes, les plus souvent évoquées par les Touaregs nomades, sont les "séances pour les génies », au cours desquelles on joue l'anzad ou le tindi (avec ou sans participation vocale) pour guérir une personne malade, c'est à dire pour «chasser les génies de son corps ", et les fêtes (baptême, mariage) qui voient les hommes s'exhiber sur leur chameau préféré pour effectuer une ronde de chameaux. (Borel 1997 : 245)

\begin{tabular}{|l|l|}
\hline Rohé Yedjulel & Je suis entré en transe \\
\hline Rohé yakh tanham & Je ne me reconnais plus \\
\hline Eias imgharen & Les sages s'en sont aperçus comme moi \\
\hline Tadjullel Hiba & Ma sœur Hiba ne se reconnaît plus \\
\hline Tanwet Baba & Elle a été frappée (tanwet) \\
\hline
\end{tabular}
terme arabe qui veut dire « âme ».

Dans le pays touareg voisin des Kel Ahaggar, le Tassili n Ajjer, des chants spécifiques et rythmes de tindi sont réservés aux génies; ils sont d'ailleurs appelés tindi rohé, le «tindi des esprits ». L'auteur anonyme qui a transcrit ce chant a traduit d'une manière approximative le terme «rohé » par «transe»; rohé dérive vraisemblablement de roh,

Ce chant de tindi recueilli à Djanet chez les Touaregs Ajjer illustre notre propos :

L'existence de tels chants spécifiques destinés aux génies prouve bien que, partout, les Touaregs organisent des cérémonies magico-thérapeutiques à base de chants et de danse. De même que dans l'Aïr, la musique et les chants offrent un espace de communion et un espace thérapeutique.

Certains devins (egahanen) ont le pouvoir de distinguer les Kel essuf noirs des Kel essuf blancs. Les techniques de divination sont variées car, s'il y a bien possession par un Kel essuf, la nature du génie impose le rituel, même si ce dernier n'est pas spécifiquement nommé. Si c'est un Kel essuf blanc (ellelen, libre), on dirige la personne possédée vers le tindi; c'est alors uniquement les hommes libres qui font une danse pour lui. Si c'est un Kel essuf «mélangé », mixte, tout le monde danse pour lui (libres et esclaves). Si c'est un Kel essuf noir (akli : esclave), seuls les iklan dansent. La danse des iklan est une danse debout, et ce sont des femmes possédées qui pratiquent une danse assise au son du tindi. Les chants consacrés aux génies ne sont pas connus de tous, malgré la transmission des répertoires des chants aux classes serviles : « les nobles conservant pour elles-mêmes les chants consacrés aux génies » (Borel $1981: 114)$.

Il existerait d'autres couleurs de génies chez les Touaregs (Rasmussen 1995); les génies bleus, proches des noirs, et les génies rouges. La possession par les génies rouges est traitée par les marabouts. Dans un monde parallèle invisible, la hiérarchie sociale se maintient ainsi sous une forme symbolique. 


\section{La tazengharet} simples motifs de réjouissance. Elle se distingue par l'absence d'instruments de musique. En effet, la composition musicale de la tazengharet consiste en un chant de femme en solo. $\mathrm{Au}$ cours du même chant, plusieurs solistes peuvent chanter en alternance ou en même temps des parties mélodiques distinctes. Le solo s'élève dans un registre aigu, accompagné de cris et de battements de mains ininterrompus, ce qui rend le texte chanté difficilement compréhensible. La cérémonie dure alors jusqu'à l'aube, ne s'arrêtant qu'après les trois chutes rituelles des imegullen, les «adeptes " possédés. Le rituel est exécuté autour d'une victime possédée par les Kel essuf, laquelle, dans la plupart des cas, a perdu l'usage de la parole et se trouve désocialisée. touchée par les génies. Les données ethnographiques soutiennent largement l'hypothèse qu'il s'agit d'une scène rituelle de possession, par la relation qu'entretiennent les rites avec la présence des Kel essuf dans le corps des adeptes. Son originalité réside dans le fait que ce rituel ne nécessite pas de sacrifice ni de musique instrumentale, éléments que les spécialistes de la possession avaient souvent jugés indispensables. La musique est pourtant là, mélodique : il y a les chants, la chorégraphie des danses, les cris, la gestuelle, les odeurs, l'aspersion d'eau, les trois chutes rituelles successives pour faire boire le possédé. Ahaggar, du fait que le répertoire spécifique pour les génies semble ne plus être maitrisé. En revanche, la tazengharet est fréquente pour des raisons curatives, dans les cas d'exorcisme. Mais elle demeure avant tout une occasion de réjouissance, car c'est aussi le 
lieu où, de par la nature très agitée de la danse, les corps se libèrent. Elle est très proche d'un autre rituel dansé, l'iswat, plus connu au Niger, et d'une autre danse en Ahaggar appelé la tehigelt.

41 La tazengharet, revendiquée par tous les Kel Ahaggar, mais pratiquée par une seule catégorie de la population, n'incluait pas les nobles et se limitait à la seule catégorie anciennement asservie des iklan. Elle est également adoptée par les harâtîn (cultivateurs noirs) après leur implantation en Ahaggar et, actuellement, même par d'autres catégories sociales. Très souvent, dans certains quartiers de Tamanrasset, des tazengharet sont animées par des enaden (forgerons) à Sorro 1 mâalmin (quartiers des forgerons) ou encore auprès des izzeggaren (cultivateurs noirs) dans le quartier d'Ankouf.

Les iklan originaires d'Afrique noire subsaharienne ont apporté avec eux des croyances et des pratiques religieuses antérieures à l'islam. Ils prirent comme modèle la société touarègue qui possède elle-même une cosmogonie particulière. En intégrant la culture nomade, l'islam s'est greffé progressivement sur ces anciennes croyances pour former un ensemble religieux original (Gast 1985 : 370).

L'islam mystique a profondément incorporé la culture locale touarègue, d'une manière assimilable à une forme de syncrétisme, terme qui peut s'adapter à ce cas; « mais sans explication, il risque de porter à confusion» (Bastide 1955 : 500). En ce qui concerne la pénétration de l'islam, il serait préférable de parler d'incorporation et d'interpénétration. "Il ne suffit pas de dire "syncrétisme" pour rendre compte de la complexité des phénomènes de métissage culturel, il faut encore montrer comment fonctionne concrètement l'interpénétration des cultures, c'est-à-dire décrire quels sont les principes à l'œuvre qui permettent cette interpénétration » (Cuche 1994).

L'Islam n'a pas cherché à enrayer complètement les usages séculaires; il a tenté de les niveler et parfois de les incorporer. C'est ainsi que des saints musulmans arrivés en Ahaggar ont investi des lieux qui étaient déjà sacrés et y ont institué quelquefois une zaouïa. De multiples exemples témoignent sur tous les continents de la vitalité des cultes de possession, de leur force d'attraction et de leur surprenante capacité d'évolution au fil des changements historiques et des déboires humains.

À Tamanrasset, il existe d'autres genres musicaux se rapportant à l'exorcisme, mais aussi d'autres pratiques en relation avec le monde invisible relevant du domaine religieux. Pour ce qui est de la musique curative, c'est par exemple le rôle spécifique de la société des qarqabou ${ }^{2}$ (crotales ou castagnettes en fer) qui pratique le dirani pour chasser et exorciser les démons. Cette tradition semble venir d'In Salah (Touat, Tidikelt) ; inconnue au Sud du Sahara, elle est différente des musiques du Nord (gnawa, aissawa) qui emploient aussi les mêmes crotales. 
Fig. 3 : Khaoulen, du groupe des enaden (forgerons), jouant des airs d'imzad accompagnant la danse d'un jeune en état de transe lors d'une tazengharet. Ziara de Tarhananet, Ahaggar.

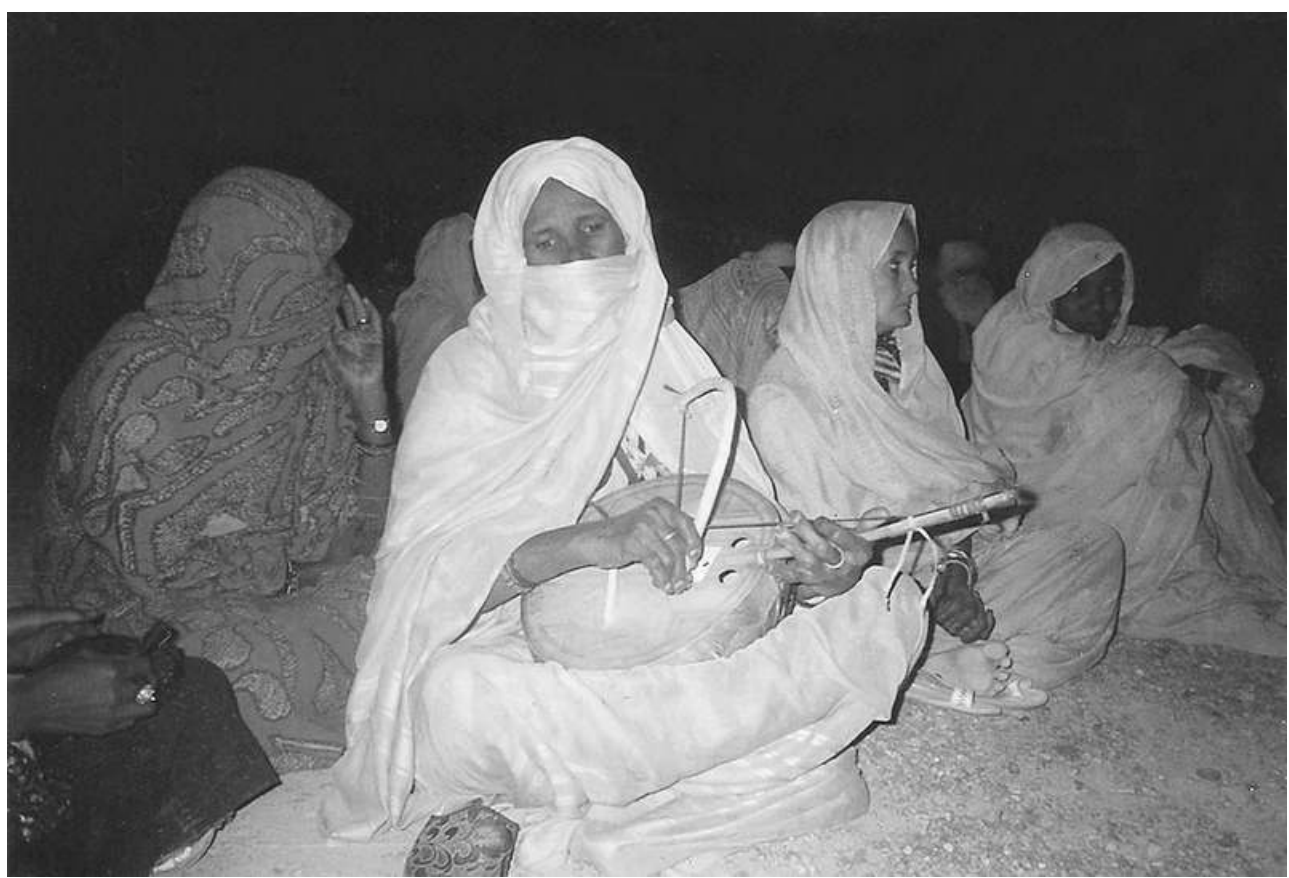

Photo Faiza Seddik-Arkam, juillet 1998.

Les rituels liés aux Kel essuf ne sont pas toujours mêlés à ceux qu'exige la religion musulmane; ils renvoient à des pratiques ancestrales parfois très éloignées de l'islam. Mais il n'empêche qu'une sorte d'allégeance se trouve dans le dispositif et dans les pratiques du culte par rapport à l'islam. Au cours du chant, on cite le nom d'Allah, ainsi que certains noms de la tradition musulmane. Mais la subversion est telle qu'elle s'attaque aussi à l'ordre religieux; les génies invoqués, les Kel essuf, sont très souvent regardés comme des entités préislamiques et "sauvages " en opposition aux aljinnan (djinns) de l'Islam pris en charge par les marabouts et les lettrés, en référence au seul Coran.

$\mathrm{Au}$ cours du rituel de la tazengharet a lieu la transgression symbolique du modèle dominant : l'esclave à qui on déniait toute existence sociale se met à officier et à posséder un savoir et, de ce fait, un pouvoir qui se présente comme un «contre-pouvoir ». Dans l'espace dévolu à la possession, nobles (imuhagh), dignitaires religieux (chorfa), forgerons et artisans (enaden), et anciens esclaves, affranchis (iklan) ou cultivateurs (harâtîn), se côtoient librement. C'est l'instant où les barrières de classe et de statut s'écroulent une à une, chacun intégrant peu à peu le culte, surtout pour ce qui est de la jeunesse de l'Ahaggar, fascinée par la magie et la violence du rituel.

Les officiants comme les possédés mettent tout en œuvre pour satisfaire les Kel essuf et s'assurer ainsi leur bienveillance. Pour mettre en évidence la notion d'altérité que l'on retrouve généralement dans les rites de possession, il suffit de suivre les rites qui la mettent en scène lors d'une tazengharet. L'altérité de ce «jeu liturgique» se manifeste dans la danse, dans la théâtralisation des comportements, dans la gestuelle, les chants, les costumes et les rythmes musicaux. 

amples pour les deux sexes, voile de coton aéré blanc, indigo, noir, rouge lie de vin. Ces couleurs fondamentales comportent de nombreux symboles. C'est un ordre social: le blanc désigne les savants lettrés et les religieux (marabouts), désignés par le qualificatif $\mathrm{d}$ ' acherif; le noir est le costume des iklan, qui apportent leur force de travail ; le rouge désigne les harâtîn (les cultivateurs noirs), désignés en tamahaq par la couleur rouge izeggâren, couleur de la terre; l'indigo est enfin la couleur qui caractérise les Touaregs. Elle est appréciée par tous les nomades, de la Péninsule arabique à la Mauritanie. En plus de son effet protecteur physique et matériel sur la peau, elle possède également une valeur magique et symbolique. Ainsi le voile touareg masculin (tagelmust), associé aux valeurs guerrières, à la galanterie et à une invocation religieuse, se trouve sacralisé. le mâalem (arabe), qui s'appelle enad (forgeron) chez les Touaregs, qui entre en scène. Son rôle dans la mise à mort sacrificielle lors des cérémonies rituelles touarègues lui donne le statut particulier d'être intermédiaire. Ce maâlem formera un cercle autour de l'adepte tombé à terre ; avec la pointe d'une épée ou d'un objet en fer, il essayera de dénouer ses mains - le fer est connu pour avoir la propriété d'éloigner les génies. Un moment est nécessaire pour que la personne tombée à terre se réveille et recommence à danser. En tant que forme enveloppante, tel un circuit fermé, le cercle est un symbole de protection assurée dans ses limites, d'où l'usage magique du cercle comme cordon de défense. Ce cercle paraît symboliser l'espace matriciel de la vie, en attente de la puissance génésique. Le geste qui consiste à jeter de l'eau à l'intérieur de ce cercle est un geste fécondant.

51 Ce même geste circulaire va être exécuté par les femmes tiklatin au cours de la danse. Ces dernières, chargées d'un brasero contenant de l'encens, de la taghelbast (gomme d'adaras, Commiphora africana), dite oum e nass en arabe, vont le faire sentir en faisant balancer l'encensoir au milieu des groupes les plus agités, ceux qui portent en eux des Kel essuf déchaînés, et ceci dans le but de purifier l'aire sacrée, de chasser ces génies. C'est le musc (teidit en Ahaggar), un parfum délicat et très apprécié des nomades et utilisé pour calmer et contenter les génies dans certains cas. La nature de l'aromate utilisé (encens ou parfum) dépendra de l'état de la personne et de la nature de sa possession.

52 Si l'on considère que la tazengharet est une musique de possession, il faut admettre que ses chants sont associés aux différents génies ayant pris possession de l'âme du sujet, bien que les Touaregs refusent de nommer les génies. «Le répertoire de chants qui s'est conservé jusqu'à aujourd'hui pourrait justifier une telle explication du fait déjà que les chants sont en nombre fixe (une douzaine) et qu'ils ont chacun leur nom, leur mélodie et parfois leur pas de danses spécifiques » (Mécheri-Saada $1986: 44)^{3}$.

53 Ce répertoire spécifique, dont parle Mécheri-Saada dans son étude d'ethnomusicologie touarègue (1994), n'est pas connu de nos jours. Il n'y a aucune publication le concernant ; mais nous avons néanmoins recueilli ces dernières années des chants «tisiway» provenant de ce répertoire, auprès d'une ancienne esclave taklit $n$ Kel Ghela du nom de Tacheka. La nature de ces chants, qui feront l'objet d'une prochaine publication, témoigne bien de l'existence d'un répertoire sacré, dédié aux génies. Une étude plus approfondie apportera sûrement des éléments nouveaux sur la variété des mélodies, des rythmes et des danses qui correspondent aux génies incarnés. 
54 A certaines tazengharet observées dans un passé proche, on a vu les possédés manger du piment, se mettre du feu sur la bouche, se percer le corps d'un couteau et le ressortir sans douleur, et être capables de boire d'un coup une bouteille de parfum (bint e sudan).

Actuellement, si un possédé devient violent, des hommes essayent de le contenir et dansent avec lui sur l'aire de danse. Ils le maintiennent fermement afin de calmer ses débordements. Lorsque cet état de transe sauvage se manifeste, la furie des élus peut être contagieuse, tout le monde s'éloigne du cercle des possédés afin d'éviter que ceux-ci ne les touchent; on dit que l'aire de danse est envahie par les Kel essuf. Des rites de purification se succèdent alors : aspersion d'eau, fumée d'encens, parfums, et ce jusqu'à la chute finale. Une fois leur état "sauvage " maitrisé, ils danseront de nouveau avec beaucoup d'harmonie, avec des gestes qui se rapprochent de ceux de l'extase mystique.

La présence du sang pose le problème du rapport particulier qu'ont les Touaregs à la souillure et à l'impureté. Les plus âgés des iklan (anciens esclaves) disent que les transes étaient bien plus violentes à une époque et que les adeptes se coupaient à l'épée ou au couteau durant la danse. Le rapport au sang a dû changer sensiblement dans ces catégories sociales, par le poids symbolique et culturel des groupes dominants qu'ils ont intégrés.

La disparition de cette dimension très violente et sauvage de la danse pourrait s'expliquer par la condamnation de tous ces rituels par des notables religieux, parce qu'ils mettent en jeu des pratiques jugées païennes rappelant à juste titre celles du Bori des Haoussa voisins, ou encore celles des Gnawa marocains. Mais c'est aussi certainement dû aux valeurs culturelles des Touaregs, qui désapprouvent le manque de maitrise de soi et qui ont une aversion pour le sang, considéré comme impur.

Voilà qui expliquerait en grande partie le fait que les Touaregs délèguent la gestion des maladies accompagnée de pratiques sacrificielles à des catégories sociales inférieures, elles mêmes considérées comme impures, ou alors à des ineslemen, des personnes dont le statut religieux autorise le recours non seulement au sacrifice, mais à d'autres pratiques rituelles prohibées ou tabous au sein de la culture locale.

\section{Tacheka et la tazengharet}

59 Pour illustrer notre propos, nous avons recueilli l'histoire de vie de Tacheka, une ancienne esclave vivant dans un quartier périphérique de Tamanrasset. Elle est reconnue pour être la maîtresse de la cérémonie de possession lors d'une tazengharet organisée à cet effet. Son histoire de vie va également retracer son alliance avec l'invisible. Elle est dite carrément « habitée » (zadghent) par les génies (maskuna en arabe). Elle est crainte pour ses liens étroits avec les Kel essuf et respectée pour ses grands pouvoirs de guérison de la possession mentale. Autour d'elle se construit toute une mythologie: mon accompagnateur refusera de me suivre chez elle au crépuscule, car c'est le moment, dit-il, où elle "cause" avec ses amis les Kel essuf. Ses voisins racontent l'avoir vue se transformer en animal, avec des pieds de chèvre; ils évitent sa maison à la tombée de la nuit pendant qu'elle est en compagnie des Kel essuf, alors qu'ils sollicitent ses chants lors des tazengharet organisées pour soulager une personne possédée ou atteinte de maladie mentale.

60 Tacheka est une timazalet; elle détient la parole sacrée, elle est celle qui possède le répertoire du chant ${ }^{4}$ et elle est crainte de tous pour sa proximité avec les Kel essuf. Son 
commerce avec la surnature lui donne un pouvoir redoutable, celui d'une chamane. On dit qu'elle est timsin, imprévisible: elle peut changer brusquement d'humeur jusqu'à changer de nature, devenir autre, être en marge de l'humanité. Ses relations étroites avec les génies, sa parole transgressive, ses gestes provocateurs font d'elle une déviante, sur le plan tant social que sexuel. A titre d'exemple, certains des poèmes qu'elle va réciter avec malice devant la mine scandalisée d'un jeune Touareg «noble » qui m'accompagnait : elle les désigne elle-même par le terme de taswilt tafelghi, une poésie érotique à la limite du vulgaire, en opposition complète avec la tangalt (la poésie voilée et métaphorique des Touaregs nobles). La nature transgressive de l'officiante fait d'elle la médiatrice par excellence « apte à transcender les catégories ordinaires » (Hell 1999: 19).

Elle est celle qui arrive à faire "boire" (saswequen) ( $t$ ) un imugullen possédé, lorsque toutes les autres femmes ont échoué. C'est pour ce rôle positif de guérisseuse qu'on l'appelle également taneslemt, équivalent féminin de l'aneslem, personnage religieux qui fait souvent office de guérisseur. La taneslemt survit grâce à la solidarité villageoise et aux offrandes des malades qu'elle soigne ; ses voisines lui apportent occasionnellement un bol de mil dilué dans de l'eau, la tahejera, aliment apprécié des nomades et que l'on ne mange actuellement qu'en temps de disette.

\section{Conclusion}

Nombreuses sont les femmes touarègues à avoir vécu un épisode initiatique : toutes ont perdu un mari jeune et un, voire plusieurs enfants.

Le rôle de la femme reste central dans cette société touarègue à tradition matrilinéaire car, si les transformations sociales ont affecté l'organisation traditionnelle touarègue et du même coup le statut privilégié des femmes, leurs nouvelles alliances avec les génies réhabilitent en quelque sorte ce statut et réactualisent le mythe de l'ancêtre féminin fondateur.

64 La désintégration de la société touarègue a affecté les représentations traditionnelles. Et dans cette recherche d'équilibre face à la déstructuration progressive de leur société, un ensemble de rites se mettent en place. La société Kel Ahaggar tente de s'adapter aux nouvelles données sociales; pour cela elle a créé de nouveaux mécanismes de défense qu'offre un paysage magico-religieux et thérapeutique riche et varié. Cette situation produit un système global de guérison, dont la musique est l'un des piliers mais pas le seul.

65 Il n'est pas question d'opposer deux pôles distincts, dont l'un relèverait de la possession par le biais de la musique et l'autre du domaine exclusif de l'exorcisme religieux, comme pour le contexte marocain que décrit Hell (2002) :

Les rites de possession qui concernent ces «chamanes possédés » descendants d'esclaves gnawa, se positionnent dans une relation d'alliance avec les génies «sauvages» responsables des maladies les plus graves, alors que les rites des marabouts (religieux) se placent eux dans le registre de l'exorcisme. Ces derniers remédient à des troubles ordinaires beaucoup moins graves et qui sont produits par des entités domestiques résidant dans des espaces familiers.

66 Le schéma que j'ai observé chez les officiants touaregs et sahariens est complètement inverse, l'exorcisme s'applique non pas aux entités ordinaires et domestiques, mais aux djinns asi, opposés au mumnin (génies croyants), désignés en arabe ; c'est dire qu'il est réservé aux génies récalcitrants asi qui refusent d'entendre raison, ou de céder à la 
menace en refusant de quitter le corps de la victime. Et ce n'est qu'en dernier recours, après avoir épuisé toutes les techniques (possession par la musique, absorption de plantes, offrande sacrificielle) que l'on se dirige vers le taleb religieux, plus onéreux, censé être plus efficace car ayant à son service de puissants serviteurs invisibles nommés khudman, et parmi eux les Afarit cités dans le Coran. Des versets dits «brûlants » (Ayat el mohreka) accompagnent ainsi le rite d'exorcisme.

Ce paysage que je décris est lui-même en pleine recomposition car il affronte la modernité. Cette dernière est intégrée à ces rituels, étendant ainsi le champ symbolique qui correspond le mieux aux nouveaux besoins de la société. On peut déjà deviner les contours d'un système symbolique beaucoup plus complexe et imbriqué qu'il n'y parait ; il faudrait une approche globale afin de saisir l'ensemble des pratiques rituelles en œuvre et situer ainsi la place de chacune des représentations sociales au sein de ce système.

\section{BIBLIOGRAPHIE}

BALOUT Lionel, dir., 1959, Collections ethnographiques : Planches. Album $N^{\circ} 1$ : Touareg Ahaggar. Paris : Arts et Métiers graphiques. [Délégation générale du gouvernement en Algérie ; Musée d'ethnographie et de préhistoire du Bardo].

BASTIDE Roger, 1955, « Le principe de coupure et le comportement afro-brésilien », in : Anais do $31^{\circ}$ Congresso internacional de americanistas, Anhembi, São Paulo, vol. 1 : 493-503.

BELLIL Rachid et DIDA Badi, 1993, « Evolution de la relation entre Kel Ahaggar et Kel Adagh », in: Hélène Claudot, dir. : Le politique dans l'histoire touarègue, Cahiers de l'IREMAM 4, Aix-en-Provence : 95-110.

BERNUS Edmond, 1969, « Maladies humaines et animales chez les Touaregs sahéliens », Journal de la société des africanistes 39(1) : 111-137.

BONTE Pierre, 1986, «Introduction », in : Le Fils et le Neveu, jeux et enjeux de la parenté touarègue. Paris : MSH ; Cambridge : CUP : 1-36.

BOREL François, 1986, « La vièle, le tambour et les génies du mal », in Jacques Hainard et Roland Kaehr, dir. : Le mal et la douleur. Neuchâtel : Musée d'ethnographie : 199-205.

BOREL François, 1987, « Une tradition orale de classe chez les Touaregs du Niger », in Marguerite Schlechten, dir. : Oralité : A propos du passage de l'oral à l'écrit. Berne : Société suisse d'ethnologie : 77-99

BOREL François, 1988, « Rythmes de passage chez les Touaregs de l'Azawagh », Cahiers de musiques traditionnelles $1: 28-38$. [Dossier « De bouche à oreille »].

BOREL François, 1997, «La musique politiquement incorrecte des Touaregs », in : Pom pom pom pom : musiques et caetera. Neuchâtel : GHK, éds., Musée d'ethnographie : 243-252.

CASAJUS Dominique, 1987, La tente dans la solitude. La société et les morts chez les Touaregs Kel Ferwan. Paris : MSH ; Cambridge : CUP. 
CASAJUS Dominique, 1989, « Le poète et le silence », in: Graines de paroles. Ecrits pour Geneviève Calame-Griaule. Paris : CNRS : 283-296.

CLAUDOT-HAWAD Hélène, 1984, « Ebawel / Essuf, les notions d'intérieur et d'extérieur dans la société touarègue ", Revue de l'Occident musulman et de la Méditerranée 38 : 171-80.

CLAUDOT-HAWAD Hélène, 1986, « La conquête du vide ou la nécessité d'être nomade chez les Touaregs ", Revue de l'Occident musulman et de la Méditerranée 41- 42 : 397-412.

COLLEYN Jean-Paul, 1989, Les chemins de Nya : culte de possession au Mali. Paris : EHESS.

CUCHE Denys, 1994, « Le concept de principe et son évolution dans la pensée de Roger Bastide », in R. Laburthe-Tolra, éd. : Roger Bastide ou le Réjouissement de l'abîme. Paris : L'Harmattan : 69-83.

FOUCAULD Charles de

1951-1952 Dictionnaire touareg-français. Dialecte de l'Ahaggar. 4 vol. Paris : Imprimerie nationale.

GAST Marceau, 1968, « Usage des encens et parfums en Ahaggar », Libyca 16 :171-174.

GAST Marceau, 1975, « Modernisation et intégration. Les influences arabo-islamiques dans la société des Kel Ahaggar (Sahara algérien) », Annuaire de l'Afrique du Nord 14 : 203-219.

GAST Marceau, 1979, « Pastoralisme nomade et pouvoir : la société traditionnelle des Kel Ahaggar », in : Production pastorale et société. Paris : MSH ; Cambridge : CUP : 201-220.

GAST Marceau, 1985, «Croyances et cultures populaires au Sahara », Mythes et croyances du monde entier 2 : le monothéisme. Paris : Lidis-Brepols : 370-382.

GIBBAL Jean-Marie, 1982, Tambours d'eau. Journal et enquête sur un culte de possession au Mali occidental. Paris : Le Sycomore.

GIBBAL Jean-Marie, 1984, Guérisseurs et magiciens du Sahel. Paris : A.M. Métaillé.

HACHI Slimane, 1998, « Une approche anthropologique de l'art figuratif préhistorique d'Afrique du Nord : analyse d'une fresque de Tin Hanakaten (Tassili n Ajjer) », Etudes et documents berbères $15-16: 163-184$.

HAMÈS Constant, 2001, « L'usage talismanique du Coran », Revue de l'histoire des religions 1/2001: Les usages du Livre saint dans l'islam et le christianisme, mis en ligne le 30 décembre 2003. URL : http ://rhr.revues.org/document1018.html.

HELL Bertrand, 1997, « Les maîtres du désordre. Anthropologie de la modernité d'un culte de possession des Gnawa (Maroc) ", in : Comment peut-on être socio-anthropologue ? autour de Georges Balandier. Paris : L'Harmattan : 153-182.

HELL Bertrand, 1999, « Ouvrir toutes les portes. Le sang sacrificiel chez les Gnawa du Maroc », in : Pierre Bonte et Michel Izard, dir. : Sacrifices en Islam : espaces et temps d'un rituel. Paris : CNRS : 383-408.

HELL Bertrand, 1999, Possession et chamanisme : les maîtres du désordre. Paris : Flammarion.

HELL Bertrand, 1999, «L'Esclave et le Saint : les Gnawa et la baraka de Mouley Abdallah Ben Hsein (Maroc) », in : Pierre Centlivres, dir. : Saints, sainteté et martyre. La fabrication de l'exemplarité. Neuchâtel : Institut d'ethnologie ; Paris : MSH : 149-174

HELL Bertrand, 2002, « Honnis mais efficaces ! Inversion sociale et pouvoir thérapeutique dans le système de la possession au Maroc », in : Convocations thérapeutiques du sacré. Paris : Karthala : 247-274. 
HUREIKI Jacques, 2000, Les médecines touarègues traditionnelles : approche ethnologique. Paris : Karthala.

KHAWAD Makhmoud , 1979, « La tagdudt », Tisuraf 3 : 79-82.

MAMMERI Mouloud, 1984, L'ahellil du Gourara. Paris : éditions de la Maison des Sciences de l'Homme.

MÉCHERI-SAADA Nadia , 1994, Musique touarègue de l'Ahaggar (Sud algérien). Paris : AWALL'Harmattan.

NICOLAISEN Johannes, 1961, « Essai sur la religion et la magie touarègues », Folk (Copenhague) 3 : 113-162.

OLIVIER DE SARDAN Jean-Pierre, 1994, « Possession, affliction et folie : les ruses de la thérapisation ", L'Homme $131:$ 7-27.

OUBROU Tariq, 1998, « Le monde des djinns dans la tradition musulmane », Possessions d'hier, possession d'aujourd'hui. Le Discours psychanalytique (Revue de l'Association freudienne), octobre $1998: 369-379$

RASMUSSEN Susan J., 1992, « Rituel specialists, ambiguity and power in Tuareg society », MAN $27: 105-128$.

RASMUSSEN Susan J., 1994, «The 'head dance', contested self, and art as a balancing act in Tuareg spirit possession », Africa 64(1) : 74-98.

ROUGET Gilbert, 1990 [1980], La musique et la transe. Esquisse d'une théorie générale des relations de la musique et de la possession. Paris : Gallimard.

ROUCH Jean, 1960, La religion et la magie Songhay. Paris : PUF.

TURNER Victor Witter, 1990, Le Phénomène rituel : structure et contre-structure. Paris : PUF.

\section{NOTES}

1. «D'après les Imajeghen Kel Fadey [...], le tendé en tant qu'instrument de rythme, n'était joué autrefois que par les femmes de la classe des Imajeghen. [...] Par ailleurs, un certain nombre de chansons anciennes furent créées par des femmes "nobles", d'où l'hypothèse de l'origine "noble" du jeu du tendé et de la transmission orale du répertoire des chants aux classes serviles, les nobles conservant pour elles-mêmes les chants consacrés aux génies » (Borel 1981 : 114).

2. Marceau Gast (communication personnelle) : «La société des qarqabou est une société quasisecrète qui a des règles morales, déontologiques, un chef, une hiérarchie, qui refuse d'être marchandisée (ils ont refusé les crédits du ministère de la culture) mais qui accepte en dédommagement de leurs services de bons repas et des petits cadeaux (viande, parfums et un peu d'argent). Les qarqabous viennent à la demande des familles, jouent et dansent dans des figures précises avec une intensité progressive et exaspérée durant laquelle des gens tombent en transe. A ces patients l'on récite à l'oreille la basmallah ou on la chante en cadence, on les asperge de parfums et l'on les entoure de fumée d'encens. »

3. Dans son étude de la musique touarègue, cette auteure n'a, comme elle le dit elle-même, présenté la tazengharet que "de manière sommaire et provisoire»; son étude concerne essentiellement le tindi, les poésies de l'imzad et les chants de mariage alewen. Je n'ai pas pu obtenir de terme local se référant directement à la possession, bien qu'à ce sujet toutes les explications recourent explicitement aux Kel essuf. Dans un chant de tazengharet nommé tangela, on appelle et on invective à la fois les génies féminins de Uden, près d'Ideles, réputé pour être 
peuplée de génies, le chant dit: «tinuba, ti n Uden ûkelmet » : jeunes filles de l'Uden , "que vous soyez perdues ».

4. Extrait du chant de Tacheka recueilli en 1999 à Tamanrasset :

Ya Ilahy, idwa ramoun Avant que l'esprit ne meure

Eni Khouru Dis à Khouru :

Igan Ihemma En toi se trouve Ihemma

Ehey, emden diwan Ils sont tous rassemblés

Ya elahy, eded Boudjemâa Boudjemâa est avec eux

Ehely, Ina bamas Il dit que sa mère est morte

Iben rukud Sans qu'il y ait de danse en son honneur

Ehey, Timediwen, engun sanagh, Mes amies, avant, je savais

Oukalen many Nous avons très peur d'eux.

\section{RÉSUMÉS}

Face aux bouleversements que vivent les communautés touarègues, la musique, les fêtes rituelles autour du tindi, de la tazengharet et, plus rarement, de l'imzad semblent réunir ces populations et leur offrir un espace de "défoulement». C'est ainsi qu'elles expriment leur bonheur de se retrouver, mais aussi leurs angoisses. Le son de la tazengharet, qui rompt le silence de la nuit, et le bruit grave du tambour-mortier tindi agissent comme un aimant. Le rituel musical est un moment où se partage une même émotion. Plus les choses vont mal, plus on entend la musique qui chasse les Kel essuf, ces mauvais génies, afin de retrouver l'harmonie dans l'espace et le temps. Cette musique, traditionnellement réservée à un groupe dominant, se trouve réappropriée par l'ensemble de la société touarègue, toutes catégories confondues, qui en fait son principal vecteur culturel et identitaire. Ici peut-être plus qu'ailleurs, la musique, comme les autres arts, par sa finalité symbolique et médiatrice entre les hommes, se prête volontiers à des représentations du sacré.

\section{AUTEUR}

\section{FAIZA SEDDIK-ARKAM}

Faiza Seddik-Arkam, née à Alger en 1970, est doctorante à l'université de Franche-ComtéBesançon. Son domaine de recherche est celui de l'anthropologie religieuse et de la maladie, portant plus spécifiquement sur les différentes pratiques religieuses et thérapeutiques des Touaregs Kel Ahaggar (Sahara algérien). Elle privilégie une méthode biographique, interactive, celle des récits de vies, des principaux officiants traditionnels alliés du monde invisible. 